\title{
Direct observation
}

David F Marks

Potential competing interests: The author(s) declared that no potential competing interests exist.

Direct observation involves observing behaviour in a relevant setting, for example patients waiting for treatment in a doctor's surgery or clinic. Direct observation includes casual observation, formal observation and participant observation. However, ethical issues are raised by planned formal observational study of people who have not given informed consent to such observations. 